\title{
Uncommon but life threatening complication of the left ventricle pacemaker lead: Coronary sinus perforation
}

\author{
Elton Soydan $^{\mathrm{a}, *}$, Çağatay Engin ${ }^{\mathrm{b}}$, Mehdi Zoghi ${ }^{\mathrm{a}}$ \\ a Ege University Medical School, Department of Cardiology, Izmir, Turkey \\ b Ege University Medical School, Department of Cardiovascular Surgery, Turkey
}

\section{Introduction}

In the presence of tricuspid valve intervention, implantation of the right ventricle lead can be hazardous for tricuspid valve function and can lead to regurgitation. Few cases have been reported with successful left ventricular pacing via the coronary sinus (CS) after tricuspid valve replacement. We represent a 43 year old female patient who had implanted a DDD-R permanent pacemaker via the CS due to symptomatic bradyarrhythmia soon after triple (aortic, mitral and tricuspid) valve stenosis 6 years ago. We aimed to highlight an uncommon and life threating complication lately diagnosed as CS perforation caused by the CS pacemaker lead.

\section{Case report}

A 43 year old female patient was referred to our hospital for permanent pacemaker control. Interrogation of pacemaker parameters showed that the battery was in the Elective Replacement Interval and a battery replacement procedure was decided. Ventricular pacing was found as $10 \%$. She had not any palpitations, lightheadedness or shortness of breath. Interestingly 6 years ago she had undergone a triple valve replacement surgery due to severe rheumatic aortic, mitral and tricuspid stenosis. Early in the postoperative period symptomatic bradyarrhythmia necessitated successful implantation of a dual chamber permanent pacemaker with the left ventricle lead implanted into the coronary sinus due to bioprosthetic tricuspid valve. During 6 year of follow up patient had no complaints at all. After hospital admission patient was transferred to the angiography laboratory and battery replacement was successfully performed with no complications. During replacement of the battery, the ventricular lead was connected to the programmer and a very high pacing threshold $(7,5 \mathrm{~V} / 1,5 \mathrm{~ms})$ with no capture even in both bipolar and unipolar mode was noticed. Fortunately the patient was not pacemaker dependent at that time with a normal sinus rhythm of 60 beats per minute. Flouroscopic view showed a laterally extended tip of the CS lead (Fig. 1). In order to counterbalance the need for pacemaker in the future and a high probability for coronary

\footnotetext{
* Corresponding author at: Ege University Medical School, Cardiology Department Erzene Mah. Gençlik Caddesi, 35040 Bornova, Izmir, Turkey.

E-mail address: elton.soydan@ege.edu.tr (E. Soydan).

Peer review under responsibility of The Society of Cardiovascular Academy.
}

sinus lead malfunction an epicardial lead implantation was decided. During the operation the apex of the heart was reached by an anterolateral thoracotomy approach with a small incision in the left side of the 5th intercostal space. Astonishingly a pacemaker lead floating freely in the pericardial space was noticed (Fig. 2). This appeared to be coronary sinus perforation due to lead manipulation during implantation and eventually explained the high threshold and capture failure of the pacemaker. The coronary sinus lead was cut from the tip and covered up with a plastic cap. The epicardial lead was adequately implanted into the apex of the heart with normal pacing parameters with pacing threshold: 0,5 V/0,40 ms. After two days of medical treatment patient was healthily discharged.

\section{Discussion}

Tricuspid valve intervention is an important challenge for the right ventricle (RV) lead implantation as excessive attempt can lead to tricuspid leaflet damage and consequently to variable extent of regurgitation. ${ }^{1}$ Even though there are some cases with implantation of RV pacing lead through the prosthetic tricuspid valve, one of the leaflet anatomic structure and movement can be damaged and eventually thrombus formation, regurgitation and perhaps infective endocarditis

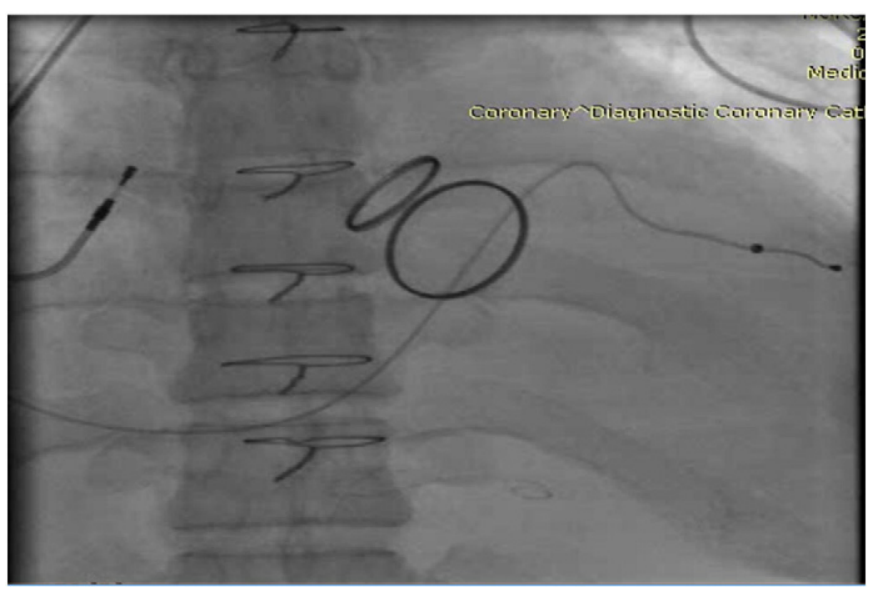

Fig. 1. Flouroscopic view of laterally extended tip of the coronary sinus lead. 


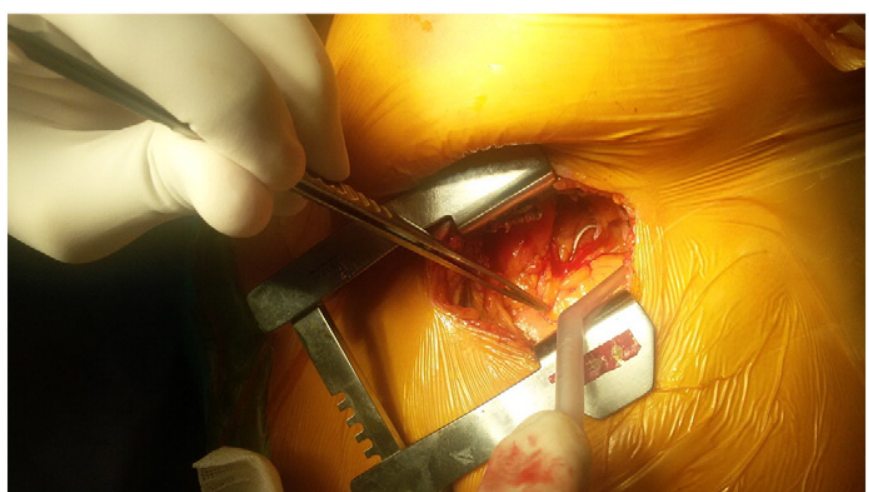

Fig. 2. Anterolateral thoracotomy approach, small incision in the left 5-th intercostal space At the apex of the heart coronary sinus perforation due to coronary sinus lead is noticed.

can occur. ${ }^{2}$ Other ways for lead implantation have been attempted. Epicardial lead implantation has its disadvantages because of being a surgical procedure perhaps not amenable for patients with comorbidities and higher threshold levels than their endocardial counterparts makes this procedure a less preferred one. ${ }^{3}$ Metallic or bio prosthetic tricuspid valves can damage the RV lead leading to pacing problems by entrapping it into the metallic frame or leaflet of the prosthetic valve. From the other hand coronary sinus access for pacing has emerged as a feasible way for ventricle pacing in patients with inaccessible RV. Tranvenous CS lead implantation was first described by Anagnostopoulos et al. as a new and suitable way for ventricular pacing in patients with mechanical tricuspid valve. ${ }^{4}$ As we look at the literature there are case studies showing successful lead implantation with stable pacing parameters in prosthetic tricuspid valve patients. ${ }^{2}$ However due to anatomical difficulties and lack of active fixation with CS leads, lead stability has been a concern. ${ }^{5}$ Nonetheless Sideris et al. and Noheria et al. demonstrated in their long term ( 2 year and 5 year period respectively) retrospective studies that CS lead implantation was safe without any threshold, dislodgement problem. ${ }^{6,7}$ Our case is unique in that there was a malfunction of the CS lead with high threshold, loss of capture after 6 years of implantation. Surprisingly the cause was coronary sinus perforation by the CS lead with no hemodynamic deterioration of the patient at all. To our knowledge this is the first described complication of a CS lead implantation in an asymptomatic patient with bioprosthetic tricuspid valve. CS perforation is a rare catastrophic complication that can result in cardiac tamponade and acute clinical deterioration. H. Nagele et al. reported only one CS perforation case in their 8 year period study with 500 patients. $^{8}$ A late and quite incidental diagnosis of this serious complication makes this case unique emphasizing that CS perforation can occur even in the late period.

\section{Conclusion}

Coronary sinus lead implantation is a relatively safe and feasible way for ventricular pacing in inaccessible RV. CS perforation should be considered if any ventricular pacing threshold or capture problem is encountered even in an asymptomatic patient.

\section{Disclosure of conflict of interest}

We do not have any conflict of interest.

\section{References}

1. Polewczyk A, Kutarski A, Tomaszewski A, Brzozowski W, Czajkowski M, Polewczyk M, Janion M. Lead dependent tricuspid dysfunction: analysis of the mechanism and management in patients referred for transvenous lead extraction. Cardiol J 2013;20: 402-410.

2. Vijayakumar M, Kamath P, Pai PG. Permanent pacing in a patient with tricuspid prosthesis-widening therapeutic use of coronary sinus. Indian Heart J 2013;65: 611-613.

3. Costa R, Scanavacca M, da Silva KR, Martinelli Filho M, Carrillo R. Novel approach to epicardial pacemaker implantation in patients with limited venous access. Heart Rhythm 2013;10:1646-1652.

4. Anagnostopoulos CE, Patel B, Fenn JE, Stansel Jr HC. Transvenous coronary sinus pacemaker. A new primary approach to heart block in patients with tricuspid prostheses. Ann Thorac Surg 1970:9:248-252.

5. Leon AR, Abraham WT, Curtis AB, et al. Safety of transvenous cardiac resynchronization system implantation in patients with chronic heart failure: combined results of over 2,000 patients from a multicenter study program. I Am Coll Cardiol 2005;46: 2348-2356.

6. Sideris Skevos, Drakopoulou Maria, Oikonomopoulos George, et al. Left ventricular pacing through coronary sinus is feasible and safe for patients with prior tricuspid valve intervention. Pacing Clin Electrophysiol 2016 Apr;39(4):378-381.

7. Noheria Amit, van Zyl Martin, Scott Luis R, et al. Single-site ventricular pacing via the coronary sinus in patients with tricuspid valve disease. Europace 2017;00:1-7.

8. Nägele $\mathrm{H}$, Behrens S, Azizi M. What can happen during coronary sinus lead implantation: dislocation, perforation and other catastrophes. Herzschrittmacherther Elektrophysiol 2007;18:243-249. 